\title{
Complete genome sequence of Mycobacterium sp. strain (Spyr1) and reclassification to Mycobacterium gilvum Spyr1
}

\author{
Aristeidis Kallimanis ${ }^{1}$, Eugenia Karabika ${ }^{1}$, Kostantinos Mavromatis ${ }^{2}$, Alla Lapidus ${ }^{2}$, Kurt M. \\ LaButti $^{2}$, Konstantinos Liolios ${ }^{2}$, Natalia Ivanova ${ }^{2}$, Lynne Goodwin ${ }^{2,3}$, Tanja Woyke ${ }^{2}$, Athana- \\ sios D. Velentzas ${ }^{4}$, Angelos Perisynakis ${ }^{1}$, Christos C. Ouzounis ${ }^{5 \S}$, Nikos C. Kyrpides ${ }^{2}$, Anna I. \\ Koukkou $^{1 *}$, and Constantin Drainas ${ }^{1+}$ \\ ${ }^{1}$ Sector of Organic Chemistry and Biochemistry, University of Ioannina, 45110 Ioannina, \\ Greece \\ ${ }^{2}$ DOE Joint Genome Institute, Walnut Creek, California, USA \\ ${ }^{3}$ Los Alamos National Laboratory, Bioscience Division, Los Alamos, New Mexico, USA \\ ${ }^{4}$ Department of Cell Biology and Biophysics, Faculty of Biology, University of Athens, \\ 15701, Athens, Greece \\ ${ }^{5}$ Centre for Bioinformatics, Department of Informatics, School of Natural \& Mathematical \\ Sciences, King's College London (KCL), London WC2R 2LS, UK \\ $\S$ Present address: Computational Genomics Unit, Institute of Agrobiotechnology, Center for \\ Research \& Technology Hellas (CERTH), GR-57001 Thessaloniki, Greece \& Donnelly Cen- \\ tre for Cellular \& Biomolecular Research, University of Toronto, 160 College Street, To- \\ ronto, Ontario M5S 3E1, Canada \\ *Corresponding author: Anna I. Koukkou, email: akukku@cc.uoi.gr \\ $\dagger$ In memory of professor Constantin Drainas who lost his life in a car accident on July 5th, 2011.
}

Mycobacterium sp.Spyr1 is a newly isolated strain that occurs in a creosote contaminated site in Greece. It was isolated by an enrichment method using pyrene as sole carbon and energy source and is capable of degrading a wide range of $\mathrm{PAH}$ substrates including pyrene, fluoranthene, fluorene, anthracene and acenapthene. Here we describe the genomic features of this organism, together with the complete sequence and annotation. The genome consists of a $5,547,747$ bp chromosome and two plasmids, a larger and a smaller one with sizes of 211,864 and 23,681 bp, respectively. In total, 5,588 genes were predicted and annotated.

Keywords: Mycobacterium gilvum, PAH biodegradation, pyrene degradation.

\section{Introduction}

Strain Spyr1 (=LMG 24558, =DSM 45189) is a new strain which based on its morphological and genomic features, belongs to the genus Mycobacterium [1]. It was isolated from Perivleptos, a creosote polluted site in Epirus, Greece $(12 \mathrm{Km}$ North of the city of Ioannina), where a wood preserving industry was operating for over 30 years. Strain Spyr1 is of particular interest because it is able to utilize a wide range of PAH substrates as sole sources of carbon and energy, including pyrene, fluoranthene, fluorene, anthracene and acenapthene. Microbial degradation is one of the major routes by which Polycyclic Aromatic Hydrocarbons (PAHs) can be removed from the environment. Strain Spyr1 metabolizes pyrene to 1-Hydroxy-2naphthoic acid which subsequently is degraded via $o$-phthalic acid, a pathway also proposed for other
Mycobacterium strains [1] exhibiting desirable PAH degradation properties as follows. Complete degradation of pyrene at concentrations $80 \mathrm{mg} / \mathrm{L} \mathrm{oc-}$ curred within eight days of incubation in the dark [1]. The extrapolated degradation rate for the growth-phase can be averaged to 10 gml $^{-1}$ day $^{-1}$, a value similar to that reported for other Mycobacterium species [2,3]. Addition of vitamins or trace amounts of yeast extract were not required for the growth of Spyr1 on any PAH, unlike other Mycobacterium spp. [4]. Use of free or entrapped cells of strain Spyr1 resulted in total removal of PAH from spiked soil samples [1]. Here a summary classification and a set of features for strain Spyr1, along with the description of the complete genome sequence and annotation are presented. 
Kallimanis et al.

\section{Classification and Features}

The phylogenetic tree of strain Spyr1 according to $16 \mathrm{~S}$ rDNA sequences is depicted in Figure 1.

The sequence identity of the 16S rRNA genes of strain Spyr1 to those from the two M. gilvum strains is $99 \%$, while the average nucleotide identity (ANI) [5] between strain Spyr1 and M. gilvum PYR-GCK is 98.5. This information indicates that Spyr1 is a strain of $M$ gilvum. Accordingly, we propose the renaming of the Spy1 strain to M. gilvum Spyr1. The ANI values between strain Spyr1 and other sequenced Mycobacteria are depicted in Figure 2.

Strain Spyr1 is an aerobic, non-motile rod, with a cell size of approximately 1.5-2.0 $\times 3.5-5.0 \mu \mathrm{m}$ and produces only a weakly positive result under Gram staining. (Figure 3). Colonies were slightly yellowish on Luria agar. The temperature range for growth was $4-37^{\circ} \mathrm{C}$ with optimum growth at $30-37^{\circ} \mathrm{C}$. The $\mathrm{pH}$ range was $6.5-8.5$ with optimal growth at pH 7.0-7.5. Strain Spyr1 was found to be sensitive to various antibiotics, the minimal inhibitory concentrations were reported as follows: chlorampenicol $10 \mathrm{mgL}^{-1}$, erythromycin $10 \mathrm{mgL}^{-1}$, rifampicin $10 \mathrm{mgL}^{-1}$ and tetracycline $10 \mathrm{mgL}^{-1}$.

Catalase and nitrate reductase tests were positive, whereas arginine dihydrolase, gelatinase, lipase, lysine and ornithine decarboxylase, oxidase, urease, citrate assimilation and $\mathrm{H}_{2} \mathrm{~S}$ production tests were negative. No acid was produced in the presence of glucose, lactose, sucrose, arabinose, galactose, glycerol, myo-inositol, maltose, mannitol, raffinose, sorbitol, sucrose, trehalose and xylose (see also Table 1).

\section{Chemotaxonomy}

Strain Spyr1 major fatty acids are $\mathrm{C}_{16: 1}(16.7 \%)$, $\mathrm{C}_{16: 0}(32,9 \%), \mathrm{C}_{18: 1}(47.5 \%), \mathrm{C}_{18: 0}(1.0 \%)$ and $\mathrm{C}_{19: 0}$ cyclo(1.1\%). The major phospholipids were phosphatidylethanolamine (PE), phosphatidylglycerol (PG) and diphospatidylglycerol (DPG) (80.4, 4.7 and $15.0 \%$ respectively).

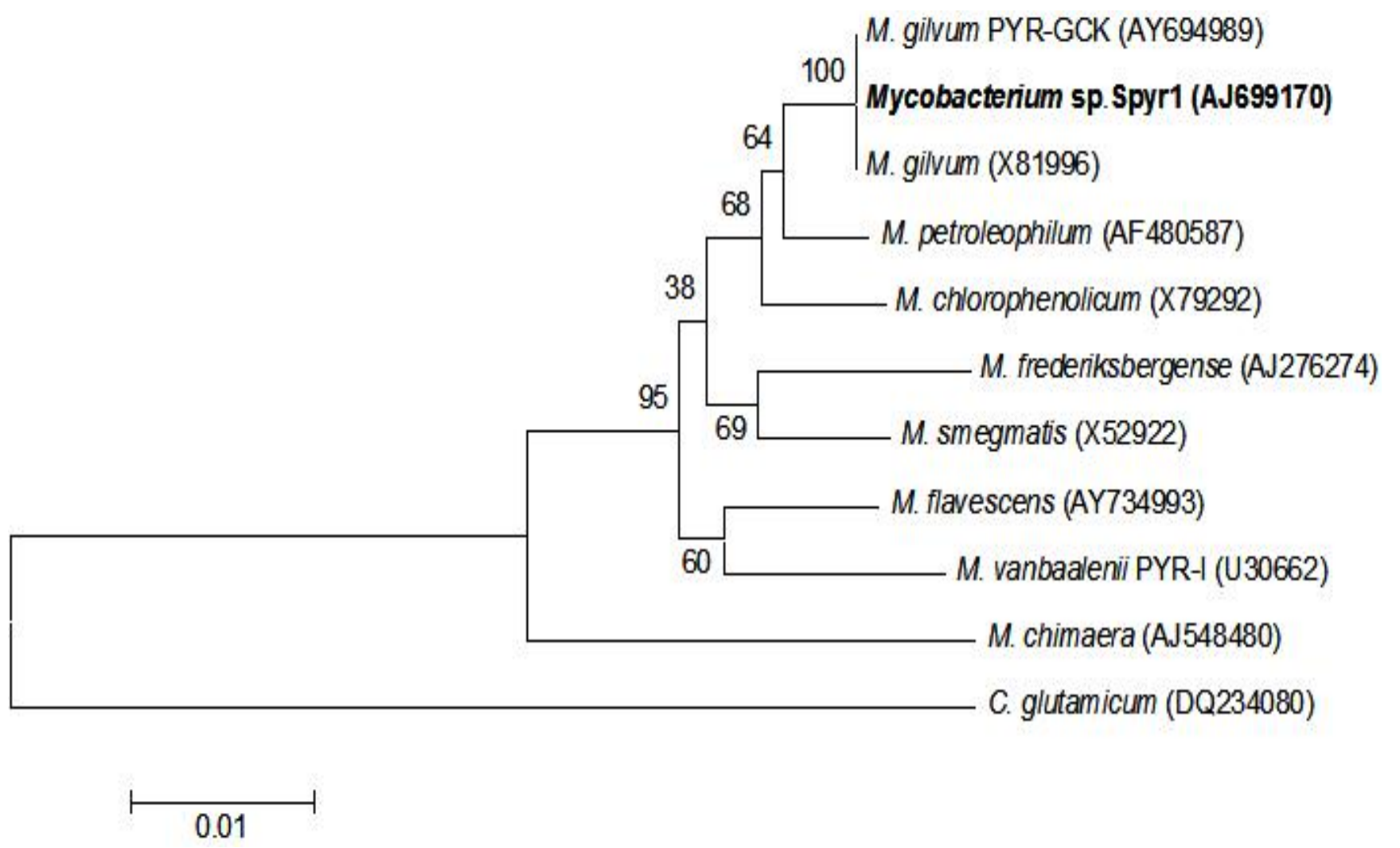

Figure 1. Phylogenetic location of strain Spyr1 among other Mycobacterium species. Corynebacterium glutamicum was used as the outgroup. The scale bar indicates the number of substitutions per nucleotide position (Number of bootstrap analysis: 1000). 
Table 1. Classification and general features of strain Spyr1 according to the MIGS recommendations [6]

\begin{tabular}{|c|c|c|c|}
\hline MIGS ID & Property & Term & Evidence code \\
\hline & \multirow{10}{*}{ Current classification } & Domain Bacteria & TAS [7] \\
\hline & & Phylum Actinobacteria & TAS [8] \\
\hline & & Class Actinobacteria & TAS [9] \\
\hline & & Subclass Actinobacteridae & TAS $[9,10]$ \\
\hline & & Order Actinomycetales & TAS [9-12] \\
\hline & & Suborder Corynebacterineae & TAS $[9,10]$ \\
\hline & & Family Mycobacteriaceae & TAS $[9-11,13]$ \\
\hline & & Genus Mycobacterium & TAS $[11,14,15]$ \\
\hline & & Species Mycobacterium gilvum & TAS $[11,13]$ \\
\hline & & strain Spyr1 & TAS [1] \\
\hline & Gram stain & Weakly positive & TAS [1] \\
\hline & Cell shape & irregular rods & TAS [1] \\
\hline & Motility & Non motile & TAS [1] \\
\hline & Sporulation & nonsporulating & NAS \\
\hline & Temperature range & mesophile & TAS [1] \\
\hline & Optimum temperature & $30^{\circ} \mathrm{C}$ & TAS [1] \\
\hline & Salinity & normal & TAS [1] \\
\hline \multirow[t]{3}{*}{ MIGS-22 } & Oxygen requirement & aerobic & TAS [1] \\
\hline & Carbon source & $\begin{array}{l}\text { Pyrene, fluoranthene, phenanthrene, anthracene, } \\
\text { glucose, yeast extract }\end{array}$ & TAS [1] \\
\hline & Energy source & $\begin{array}{l}\text { Pyrene, fluoranthene, phenanthrene, anthracene, } \\
\text { glucose, yeast extract }\end{array}$ & TAS [1] \\
\hline MIGS-6 & Habitat & Soil & TAS [1] \\
\hline MIGS-15 & Biotic relationship & Free-living & NAS \\
\hline \multirow[t]{3}{*}{ MIGS-14 } & Pathogenicity & none & NAS \\
\hline & Biosafety level & 1 & NAS \\
\hline & Isolation & Creosote contaminated soil & TAS [1] \\
\hline MIGS-4 & Geographic location & Perivleptos, Epirus, Greece & TAS [1] \\
\hline MIGS-5 & Sample collection time & April 2000 & TAS [1] \\
\hline MIGS-4.1 & Latitude & 39.789 & NAS \\
\hline MIGS-4.2 & Longitude & 20.781 & NAS \\
\hline MIGS-4.3 & Depth & $10-20 \mathrm{~cm}$ & TAS [1] \\
\hline MIGS-4.4 & Altitude & $500 \mathrm{~m}$ & TAS [1] \\
\hline
\end{tabular}

Evidence codes - TAS: Traceable Author Statement (i.e., a direct report exists in the literature); NAS: Nontraceable Author Statement (i.e., not directly observed for the living, isolated sample, but based on a generally accepted property for the species, or anecdotal evidence). These evidence codes are from of the Gene Ontology project [16]. 
ANI between Mycobacterium sp Spyr1 and other Mycobacterla

Mycobacterium gilmm PYF-GCK Mycobacterium vanbaatenii PYR-1 Mycotacterium so KWS Mycobactarium so dis Mycobacterium sp MCS Mycobactorim smegmatis MC2 150 Mycobacterium avium 104 Mycotactonim anum paratubercusosis K-10 Mycobacterium uicerans Agysg Mycotacteritim abscessus CIP 104536 Mycobacterium bovis BCG Fastour 1173P2 Mycobacteritim bovis AFà $123 / 97$ Mycobactarium bovis BCG Tokyo 172 Mycobacterium tubercuosis CoCt55t Mycohactarum tubercudosis F11 (EXPEC) Mycobacterium tuberculosis Ha7Fv (tab strain) Mycotactorium theerculosis Ha7Pa Mycobacterium tuberculosis KZN 1435 (MDF) Mycotacteritisn marinum Mt, ATCC BAA-535 Mycobacterium tepree Er4923 Mycotactorism tepree IN

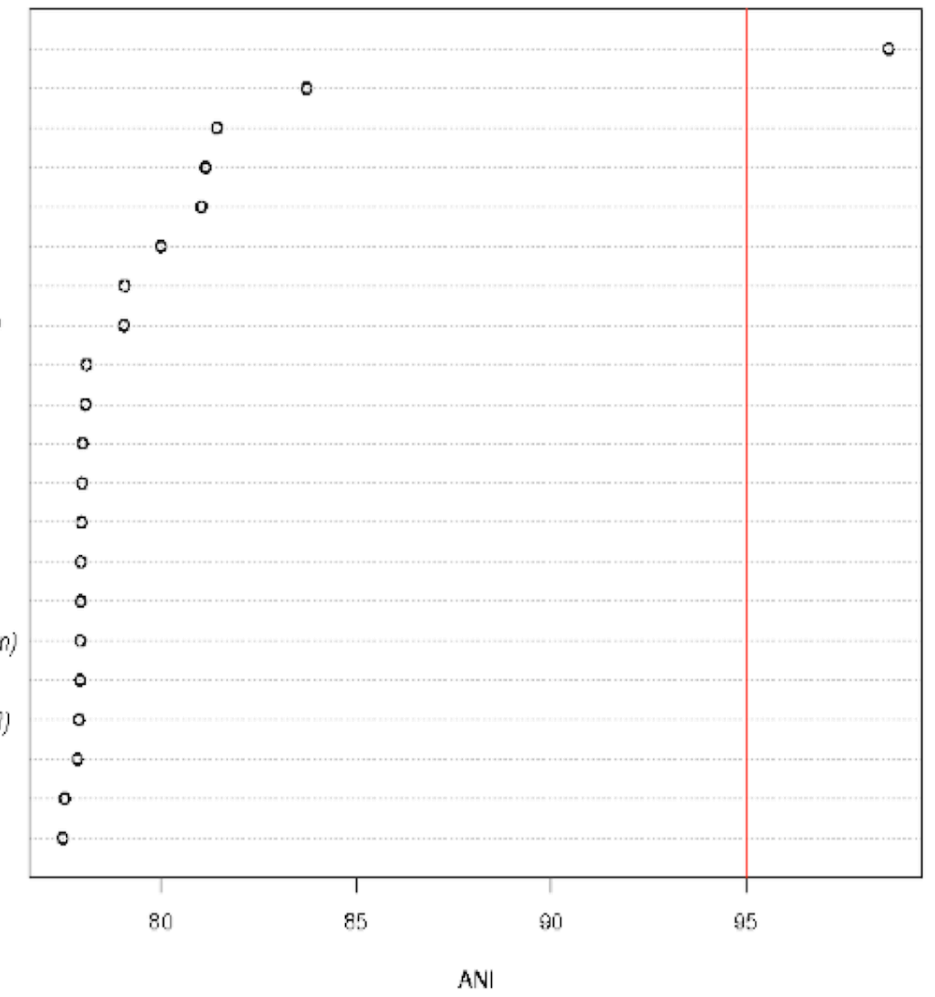

Figure 2. ANI values between Mycobacterium sp. Spyr1 and other Mycobacteria. The red line is drawn at ANI 95 a suggested threshold for species.

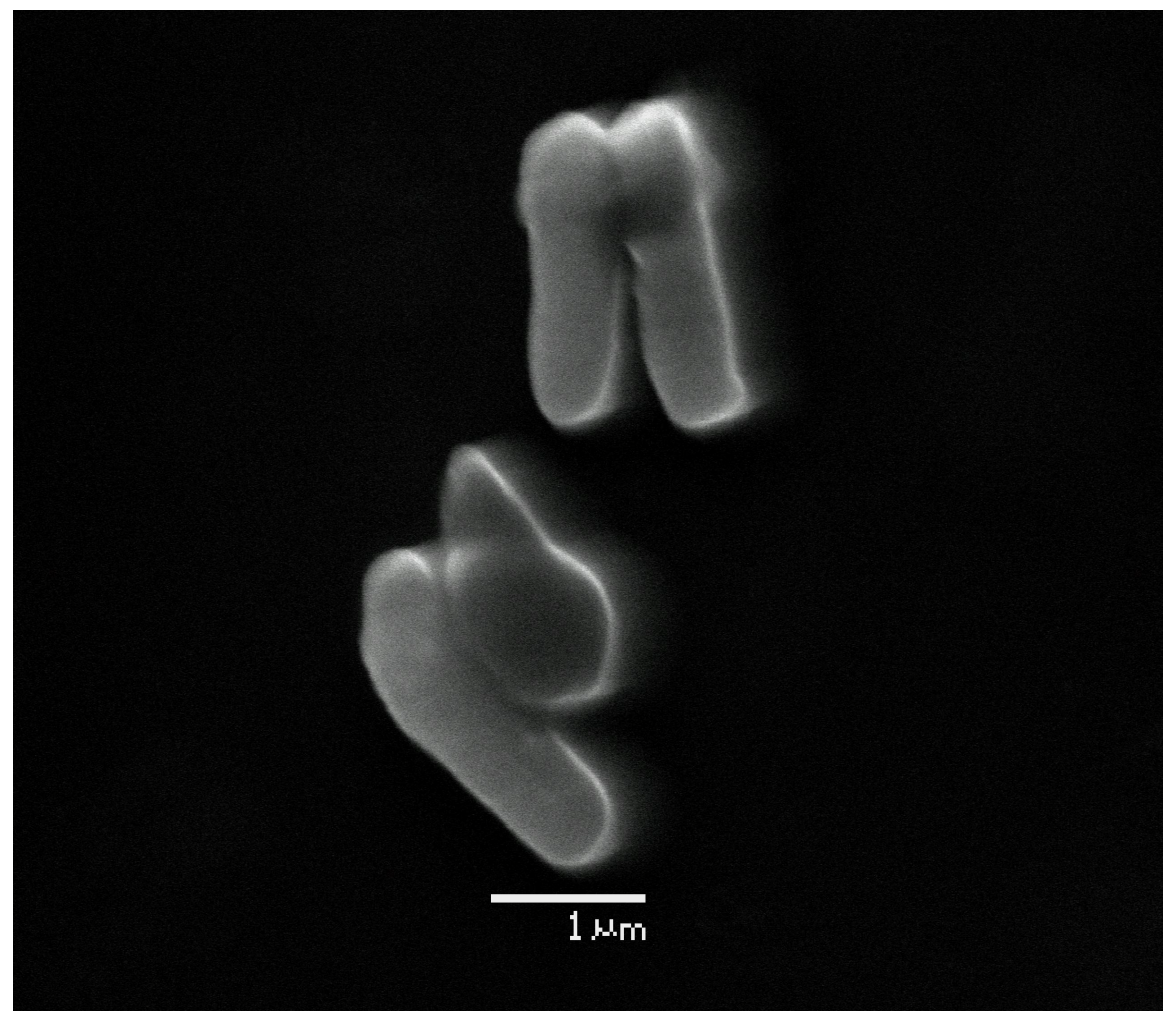

Figure 3. Scanning electron micrograph of Mycobacterium gilvum strain Spyr1. 


\section{Genome sequencing information Genome project history}

This organism was selected for sequencing on the basis of its biodegradation capabilities, i.e. metabolizes phenanthrene as a sole source of carbon and energy. The genome project is deposited in the Genome OnLine Database [17] and the com- plete genome sequence is deposited in GenBank. Sequencing, finishing and annotation were performed by the DOE Joint Genome Institute (JGI). A summary of the project information is shown in Table 2.

Table 2. Genome sequencing project information

\begin{tabular}{lll}
\hline MIGS ID & Property & Term \\
\hline MIGS-31 & Finishing quality & Finished \\
MIGS-28 & Libraries used & $\begin{array}{l}\text { Tree genomic libraries: Sanger 9 kb pMCL200, } \\
\text { fosmids and 454 standard library }\end{array}$ \\
MIGS-29 & Sequencing platforms & ABI3730, 454 GS FLX \\
MIGS-31.2 & Sequencing coverage & $10.26 \times$ Sanger; 43.3 $\times$ pyrosequence \\
MIGS-30 & Assemblers & Newbler version 1.1.02.15, Arachne \\
MIGS-32 & Gene calling method & Prodigal 1.4, GenePRIMP \\
& Genbank ID & CP002385, CP002386, CP002387 \\
& Genbank Date of Release & December 21, 2010 \\
& GOLD ID & Gc01567 \\
& NCBI project ID & 28521 \\
& Database: IMG & 649633070 \\
MIGS-13 & Source material identifier & DSM 45189 \\
& Project relevance & Bioremediation, PAH degradation \\
\hline
\end{tabular}

\section{Growth conditions and DNA isolation}

Mycobacterium gilvum Spyr1, DSM 45189 was grown aerobically at $30^{\circ} \mathrm{C}$ on MM M9 containing $0.01 \%(\mathrm{w} / \mathrm{v})$ pyrene. DNA was isolated according to the standard JGI (CA, USA) protocol for bacterial genomic DNA isolation using CTAB.

\section{Genome sequencing and assembly}

The genome of Mycobacterium gilvum Spyr1 strain was sequenced using a combination of Sanger and 454 sequencing platforms. All general aspects of library construction and sequencing can be found at the JGI website [18]. Pyrosequencing reads were assembled using the Newbler assembler version 1.1.02.15 (Roche). Large Newbler contigs were broken into 6,290 overlapping fragments of $1,000 \mathrm{bp}$ and entered into assembly as pseudoreads. The sequences were assigned quality scores based on Newbler consensus q-scores with modifications to account for overlap redundancy and to adjust inflated q-scores. A hybrid 454/Sanger assembly was made using the Arachne assembler [19]. Possible mis-assemblies were corrected and gaps between contigs were closed by editing in Consed, with custom primer walks from sub- clones or PCR products. A total of 346 Sanger finishing reads were produced to close gaps, resolve repetitive regions, and raise the quality of the finished sequence. The error rate of the completed genome sequence is less than 1 in 100,000. Together, the combination of the Sanger and 454 sequencing platforms provided $53.56 \times$ coverage of the genome. The final assembly contains 61,443 Sanger reads and 1,300,893 pyrosequencing reads.

\section{Genome annotation}

Genes were identified using Prodigal [20] as part of the Oak Ridge National Laboratory genome annotation pipeline, followed by a round of manual curation using the JGI GenePRIMP pipeline [21]. The predicted CDSs were translated and used to search the National Center for Biotechnology Information (NCBI) nonredundant database, UniProt, TIGR-Fam, Pfam, PRIAM, KEGG, COG, and InterPro databases. Comparative analysis was performed within the Integrated Microbial Genomes (IMG) platform [22]. 
Kallimanis et al.

\section{Genome properties}

The genome consists of a 5,547,747 bp long circular chromosome with a $\mathrm{G}+\mathrm{C}$ content of $68 \%$ and two plasmids (Figures 4-6, Table 3). The larger is 211,864 bp long with $66 \% \mathrm{G}+\mathrm{C}$ content and the smaller 23,681 bp with $64 \% \mathrm{G}+\mathrm{C}$ content (Table 3 and Figure 4, Figure 5 and Figure 6) Of the 5,434 genes predicted, 5,379 were protein-coding genes, and 55 RNAs; 30 pseudogenes were also identified. The majority of the protein-coding genes $(67.3 \%)$ were assigned a putative function while the remaining ones were annotated as hypothetical proteins. The distribution of genes into COGs functional categories is presented in Table 4.

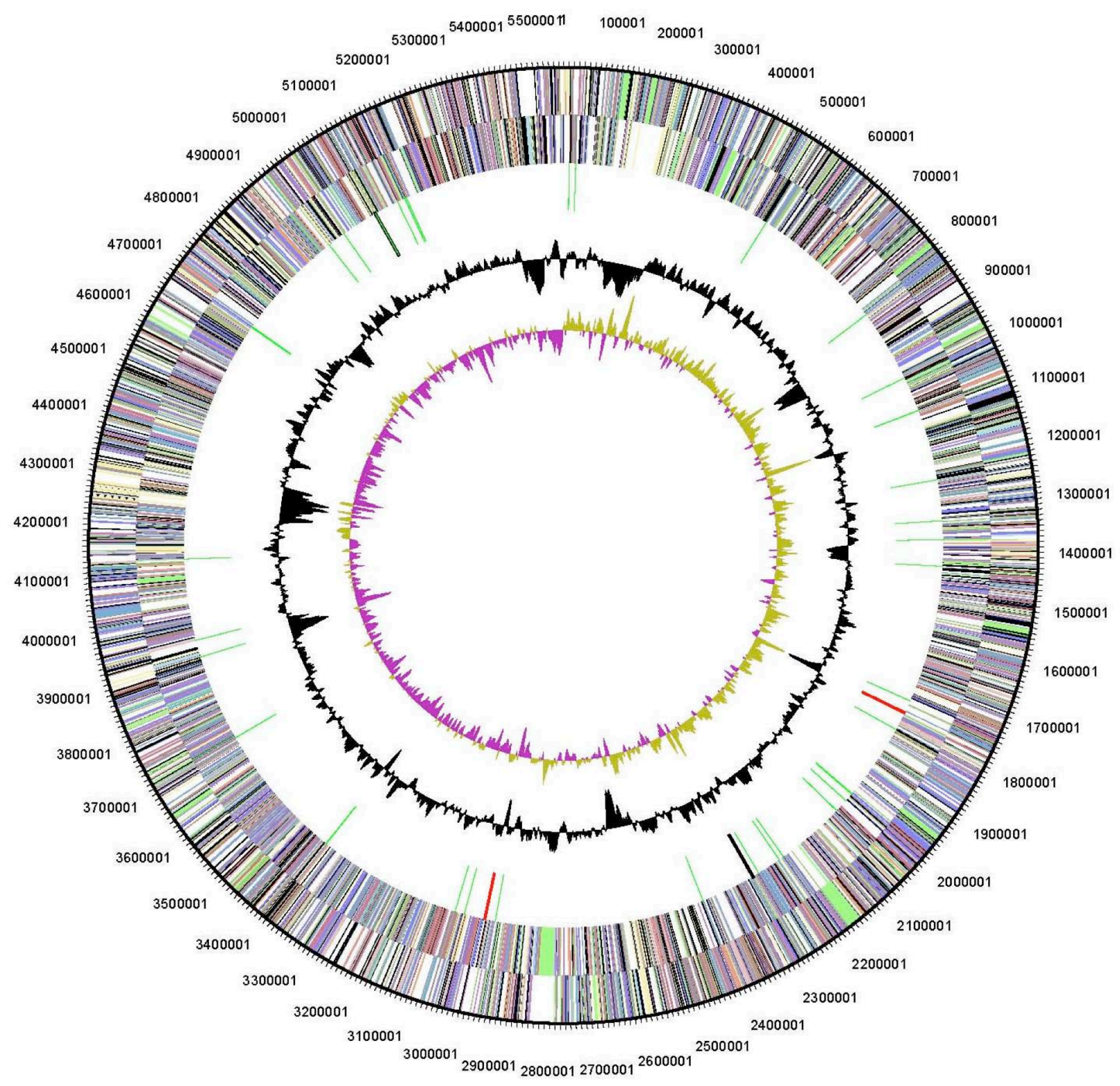

Figure 4. Graphical circular map of the chromosome of strain Spyr1. From outside to the center: Genes on forward strand (color by COG categories), Genes on reverse strand (color by COG categories), RNA genes (tRNAs green, rRNAs red, other RNAs black), GC content, GC skew. 


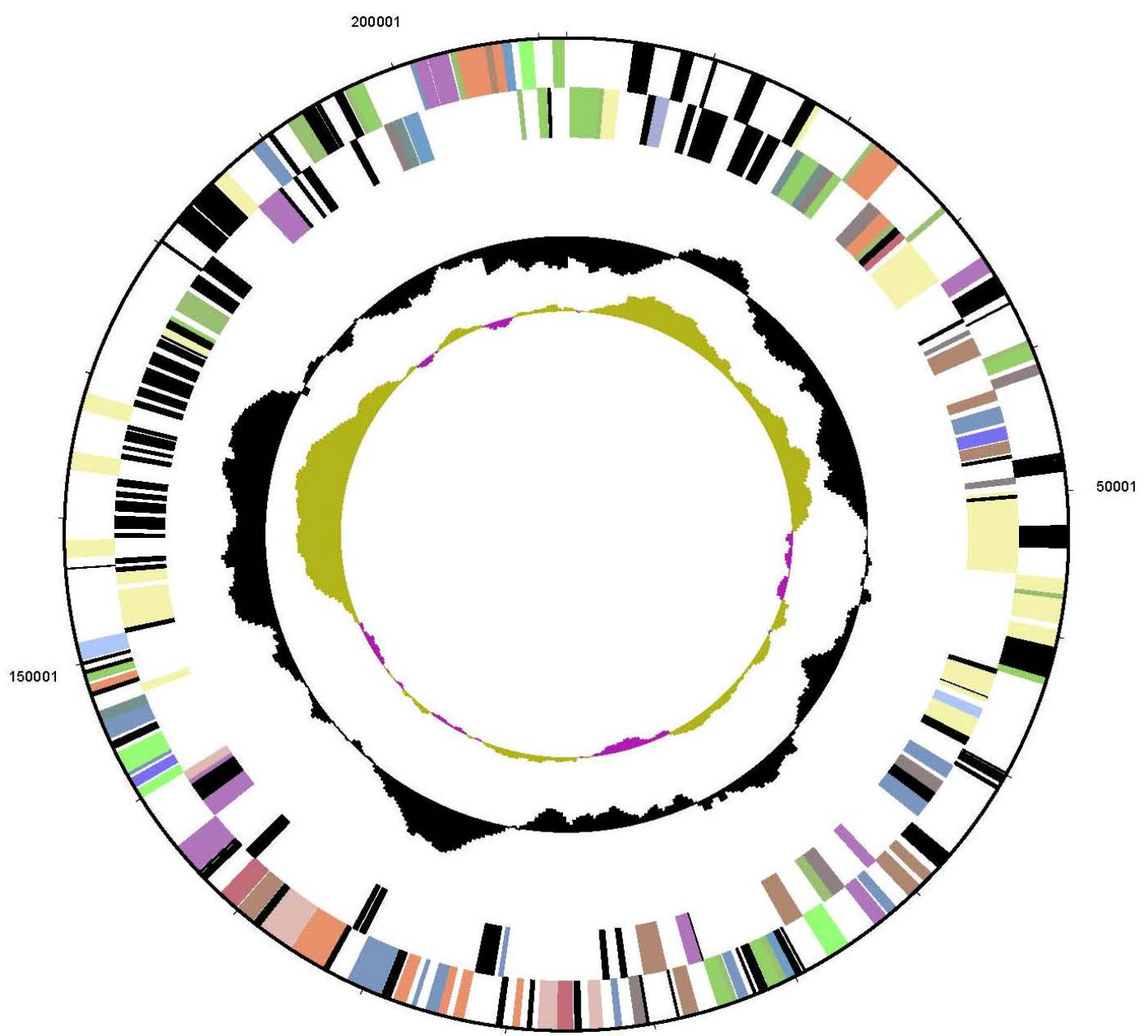

100001

Figure 5. Graphical circular map of first plasmid of strain Spyr1. From outside to the center: Genes on forward strand (color by COG categories), Genes on reverse strand (color by COG categories), RNA genes (tRNAs green, rRNAs red, other RNAs black), GC content, GC skew.

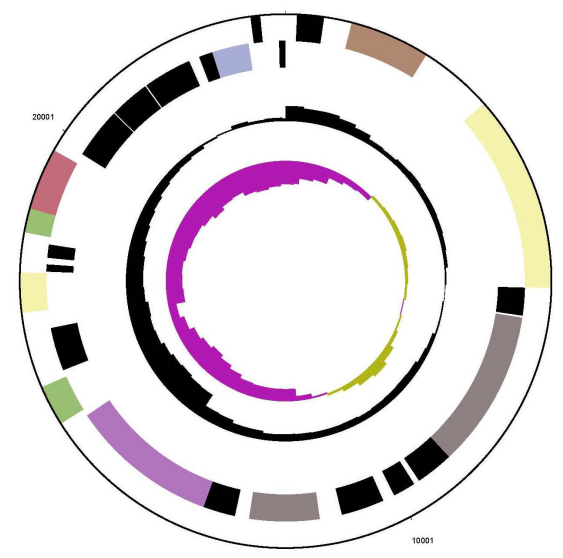

Figure 6. Graphical circular map of second plasmid of strain Spyr1. From outside to the center: Genes on forward strand (color by COG categories), Genes on reverse strand (color by COG categories), RNA genes (tRNAs green, rRNAs red, other RNAs black), GC content, GC skew. 
Table 3. Genome Statistics

\begin{tabular}{lrr}
\hline Attribute & Value & \% of Total \\
\hline Genome size (bp) & $5,783,292$ & $100.00 \%$ \\
DNA coding region (bp) & $5,256,086$ & $90.88 \%$ \\
DNA G+C content (bp) & $3,918,840$ & $67.76 \%$ \\
Number of replicons & 1 & \\
Extrachromosomal elements & 2 & \\
Total genes & 5,434 & $100.00 \%$ \\
RNA genes & 55 & $1.01 \%$ \\
rRNA operons & 2 & \\
Protein-coding genes & 5,379 & $98.99 \%$ \\
Pseudo genes & 30 & $0.55 \%$ \\
Genes with function prediction & 3,657 & $67.30 \%$ \\
Genes in paralog clusters & 403 & $7.42 \%$ \\
Genes assigned to COGs & 4,038 & $74.31 \%$ \\
Genes assigned Pfam domains & 4,188 & $77.07 \%$ \\
Genes with signal peptides & 1,617 & $29.76 \%$ \\
Genes with transmembrane helices & 1,185 & $33.80 \%$ \\
CRISPR repeats & 0 & \\
\hline
\end{tabular}

Table 4. Number of genes associated with the general COG functional categories

\begin{tabular}{crrl}
\hline Code & value & \%age & Description \\
\hline J & 154 & 3.4 & Translation, ribosomal structure and biogenesis \\
A & 20 & 0.4 & RNA processing and modification \\
K & 398 & 8.7 & Transcription \\
L & 305 & 6.7 & Replication, recombination and repair \\
B & 1 & 0.0 & Chromatin structure and dynamics \\
D & 34 & 0.7 & Cell cycle control, cell division, chromosome partitioning \\
Y & 0 & 0.0 & Nuclear structure \\
V & 46 & 1.0 & Defense mechanisms \\
T & 193 & 4.2 & Signal transduction mechanisms \\
M & 176 & 3.9 & Cell wall/membrane/envelope biogenesis \\
N & 10 & 0.2 & Cell motility \\
Z & 1 & 0.0 & Cytoskeleton \\
W & 0 & 0.0 & Extracellular structures \\
U & 38 & 0.8 & Intracellular trafficking, secretion and vesicular transport \\
O & 132 & 2.9 & Posttranslational modification, protein turnover, chaperones \\
C & 303 & 6.6 & Energy production and conversion \\
G & 198 & 4.3 & Carbohydrate transport and metabolism \\
E & 320 & 7.0 & Amino acid transport and metabolism \\
F & 81 & 1.8 & Nucleotide transport and metabolism \\
H & 170 & 3.7 & Coenzyme transport and metabolism \\
I & 412 & 9.0 & Lipid transport and metabolism \\
P & 216 & 4.7 & Inorganic ion transport and metabolism \\
Q & 362 & 7.9 & Secondary metabolites biosynthesis, transport and catabolism \\
R & 636 & 14.0 & General function prediction only \\
S & 351 & 7.7 & Function unknown \\
- & 1,396 & 25.7 & Not in COGs \\
\hline & & &
\end{tabular}




\section{Acknowledgements}

This work was funded by the program "Pythagoras II" of EPEAEK with 25\% National Funds and 75\% European Social Funds (ESF) and partly supported by the European Commission FP7 Collaborative Project MICROME (grant agreement number 222886-2). Sequencing and annotation was supported by the US Department of Energy Office of Science, Biological and Environmental

\section{References}

1. Karabika E, Kallimanis A, Dados A, Pilidis G, Drainas C, Koukkou Al. Taxonomic identification and use of free and entrapped cells of a new Mycobacterium sp., strain Spyr1 for degradation of polycyclic aromatic hydrocarbons (PAH). Appl Biochem Biotechnol 2009; 159:155-167. PubMed doi:10.1007/s12010-008-8463-1

2. Dean-Ross D, Cerniglia CE. Degradation of pyrene by Mycobacterium flavescens. Appl Microbiol Biotechnol 1996; 46:307-312. PubMed doi:10.1007/s002530050822

3. Vila J, Lopez Z, Sabate J, Minuillon C, Solanas A, Grifoll M. Identification of a novel metabolite in the degradation of pyrene by Mycobacterium sp. strain AP1: actions of the isolate on two- and three-ring polycyclic aromatic hydrocarbons. Appl Environ Microbiol 2001; 67:5497-5505. PubMed doi:10.1128/AEM.67.12.5497$\underline{5505.2001}$

4. Heitkamp MA, Franklin W, Cerniglia CE. Microbial metabolism of polycyclic aromatic hydrocarbons: isolation and characterization of a pyrenedegrading bacterium. Appl Environ Microbiol 1988; 54:2549-2555. PubMed

5. Konstantinidis KT, Tiedje JM. Genomic insights that advance the species definition for prokaryotes. Proc Natl Acad Sci USA 2005; 102:25672572. PubMed doi:10.1073/pnas.0409727102

6. Field D, Garrity G, Gray T, Morrison N, Selengut J, Sterk P, Tatusova T, Thomson N, Allen MJ, Angiuoli SV, et al. The minimum information about a genome sequence (MIGS) specification. Nat Biotechnol 2008; 26:541-547. PubMed doi:10.1038/nbt1360

7. Woese CR, Kandler O, Wheelis ML. Towards a natural system of organisms: proposal for the domains Archaea, Bacteria, and Eucarya. Proc Natl Acad Sci USA 1990; 87:4576-4579. PubMed doi:10.1073/pnas.87.12.4576

8. Garrity GM, Holt JG. The Road Map to the Manual. In: Garrity GM, Boone DR, Castenholz RW (eds), Bergey's Manual of Systematic Bacteriology,
Research Program, and by the University of California, Lawrence Berkeley National Laboratory under contract No. DE-AC02-05CH11231, Lawrence Livermore National Laboratory under Contract No. DE-AC52-07NA27344, and Los Alamos National Laboratory under contract No. DE-AC02-06NA25396.

Second Edition, Volume 1, Springer, New York, 2001, p. 119-169.

9. Stackebrandt E, Rainey FA, Ward-Rainey NL. Proposal for a new hierarchic classification system, Actinobacteria classis nov. Int I Syst Bacteriol 1997; 47:479-491. doi:10.1099/00207713-47-2$\underline{479}$

10. Zhi XY, Li WJ, Stackebrandt E. An update of the structure and 16S rRNA gene sequence-based definition of higher ranks of the class Actinobacteria, with the proposal of two new suborders and four new families and emended descriptions of the existing higher taxa. Int J Syst Evol Microbiol 2009; 59:589-608. PubMed doi:10.1099/ijs.0.65780-0

11. Skerman VBD, McGowan V, Sneath PHA. Approved Lists of Bacterial Names. Int / Syst Bacteriol 1980; 30:225-420. doi:10.1099/0020771330-1-225

12. Buchanan RE. Studies in the nomenclature and classification of bacteria. II. The primary subdivisions of the Schizomycetes. I Bacteriol 1917; 2:155-164. PubMed

13. Chester FD. Report of mycologist: bacteriological work. Delaware Agricultural Experiment Station Bulletin 1897; 9:38-145.

14. Lehmann KB, Neumann R. Atlas und Grundriss der Bakteriologie und Lehrbuch der speziellen bakteriologischen Diagnostik, First Edition, J.F. Lehmann, München, 1896, p. 1-448.

15. Runyon EH, Wayne LG, Kubica GP. Genus I. Mycobacterium Lehmann and Neumann 1896, 363. In: Buchanan RE, Gibbons NE (eds), Bergey's Manual of Determinative Bacteriology, Eighth Edition, The Williams and Wilkins Co., Baltimore, 1974, p. 682-701.

16. Ashburner M, Ball CA, Blake JA, Botstein D, Butler H, Cherry JM, Davis AP, Dolinski K, Dwight SS, Eppig JT, et al. Gene ontology: tool for the unification of biology. Nat Genet 2000; 25:25-29. $\underline{\text { PubMed doi:10.1038/75556 }}$ 
Kallimanis et al.

17. Liolios K, Chen IM, Mavromatis K, Tavernarakis $\mathrm{N}$, Hugenholtz P, Markowitz VM, Kyrpides NC. The Genomes On Line Database (GOLD) in 2009: status of genomic and metagenomic projects and their associated metadata. Nucleic Acids Res 2010; 38:D346-D354. PubMed doi:10.1093/nar/gkp848

18. The Joint Genome Institute. http://www.jgi.doe.gov

19. Batzoglou S, Jaffe DB, Stanley K, Butler J, Gnerre S, Mauceli E, Berger B, Mesirov JP, Lander ES. ARACHNE: a whole-genome shotgun assembler. Genome Res 2002; 12:177-189. PubMed doi:10.1101/gr.208902

20. Hyatt D, Chen GL, Locascio PF, Land ML, Larimer FW, Hauser LJ. Prodigal Prokaryotic Dynamic Programming Genefinding Algorithm. BMC Bioinformatics 2010; 11:119. PubMed doi:10.1186/1471-2105-11-119

21. Pati A, Ivanova N, Mikhailova N, Ovchinikova G, Hooper SD, Lykidis A, Kyrpides NC. GenePRIMP:
A Gene Prediction Improvement Pipeline for microbial genomes. Nat Methods 2010; 7:455457. PubMed doi:10.1038/nmeth.1457

22. Markowitz VM, Chen IMA, Palaniappan K, Chu K, Szeto E, Grechkin Y, Rather A, Anderson I, Lykidis A, Mavromatis K, et al. The Integrated Microbial Genomes (IMG) system: an expanding comparative analysis resource. Nucleic Acids Res 2010; 38:D382-D390. PubMed doi:10.1093/nar/gkp887

23. Pribram E. A contribution to the classification of microorganisms. J Bacteriol 1929; 18:361-394. PubMed

24. Conn HJ, Dimmick I. Soil bacteria similar in morphology to Mycobacterium and Corynebacterium. J Bacteriol 1947; 54:291-303.

25. Stanford JL, Gunthorpe WJ. A study of some fastgrowing scotochromogenic mycobacteria including species descriptions of Mycobacterium gilvum (new species) and Mycobacterium duvalii (new species). Br J Exp Pathol 1971; 52:627-637. PubMed 